\title{
Shocks and high velocity deformation
}

\author{
R CHIDAMBARAM
}

Physics Group, Bhabha Atomic Research Centre, Bombay 400085, India

\begin{abstract}
The shock Hugoniot curves of a large number of materials up to a few Mbar have been obtained experimentally. Metallurgical examination and physical measurements on metallic and other samples recovered after shock loading up to several $100 \mathrm{kbar}$ indicate the existence of large concentrations of point, line and planar defects. Dislocation mechanisms have been invoked to explain shock wave propagation and the phenomena related to the quick homogenisation of stress and strain behind the shock front. Computer simulation models using molecular dynamics calculations have also been used to understand some aspects of shock wave propagation at an atomistic level. For very strong shocks, the material is expected to melt under shock heating, but the experimental evidence regarding this is inconclusive. A combination of shock temperature measurement and theory may be able to answer this question.
\end{abstract}

Keywords. Shock propagation; shock deformation; shock melting.

\section{Introduction}

During the last decade or two, our understanding of the mechanical effects of shock waves on materials has advanced considerably. There is now a wealth of metallurgical information on samples recovered after being subjected to shock loading. Active measurements of physical parameters such as the electrical resistivity have also been attempted in a few cases. Physical phenomena like shock deformation, phase transformations under shock loading and shock melting have been understood to a somewhat smaller extent at the microscopic level. We shall discuss some of these aspects in this paper.

If the shock wave propagating in a material is considered to be a sharp discontinuity in stress, then, using the laws of conservation of mass, momentum and energy, it can be shown that the undisturbed state is related to the shocked state at the shock front as follows:

$$
\begin{aligned}
& U_{s} \rho_{0}=\rho_{1}\left(U_{s}-U_{p}\right), \\
& P_{1}-P_{0}=\rho_{0} U_{s} U_{p}, \\
& E_{1}-E_{0}=\frac{1}{2}\left(P_{1}+P_{0}\right)\left(V_{0}-V_{1}\right),
\end{aligned}
$$

where $U_{s}$ is the shock front velocity, $U_{p}$ is the particle velocity in the compressed region; $P, E, \rho$ and $V(=1 / \rho)$ are respectively the pressure, specific internal energy, density and specific volume: the suffixes 1 and 0 represent quantities in the shocked and the unshocked regions respectively.

If the equation of state $E=E(P, V)$ of the material is also known, we can use (3) to write $P$ as a function of $V$ (for all practical purposes, $P_{0} \simeq 0$ ). The locus of all states $(P, V)$ which can be obtained by a single shock from an initial state $\left(P_{0}, V_{0}\right)$ is known as the Rankine-Hugoniot curve or simply the Hugoniot curve (figure 1). 


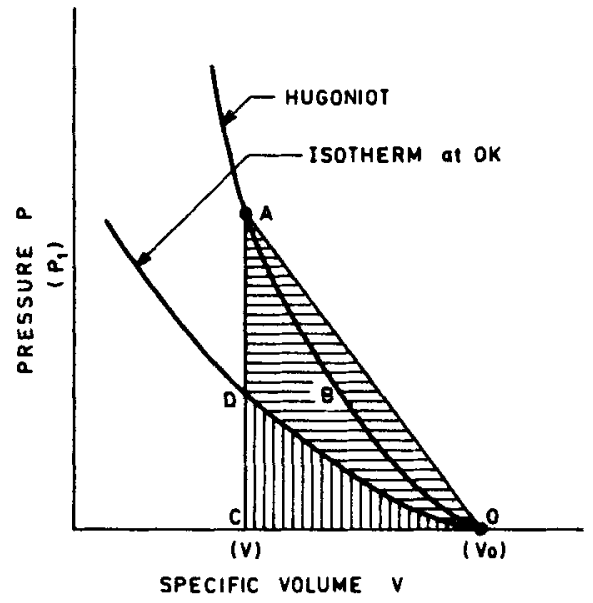

Figure 1. The Hugoniot curve. The vertically-hatched area is a measure of the cold compressive energy. The horizontally-hatched area is a measure of the heat energy in the shocked sample.

It is evident from (3) that the internal energy deposited in the compressed body is the area of the triangle $O A C$. If the compression is carried out isothermally at $0^{\circ} \mathrm{K}$ to the same final volume $V$, the material will be at the point $D$. The area of the curved triangle $O D C$ therefore represents the "cold" elastic energy. The difference between the areas of $O A C$ and $O D C$ is a measure of the heat energy deposited by the shock in the compressed sample; the contribution from defects created by the shock, such as vacancies and dislocations, is relatively negligible. To give some numbers, a shock pressure of $1 \mathrm{Mbar}^{*}$ will compress a sample of aluminium from a density of 2.78 to $4.30 \mathrm{~g} / \mathrm{cm}^{3}$ and heat it to $3540 \mathrm{~K}$. The corresponding shock velocity is $10.1 \mathrm{~mm} / \mu \mathrm{sec}$ and the particle velocity is $3.6 \mathrm{~mm} / \mu \mathrm{sec}$.

After the shock wave has passed, the sample will unload along the curved path $A B O$ doing work on the surroundings represented by the area of the curved triangle $O B A C$. (For simplicity we assume that the terminal specific volume $\simeq$ the initial value $V_{0}$, which would be true in the absence of porosity in the initial sample, etc.) The difference in the areas $O A C$ and $O B A C$ represents the (waste) heat deposited in the terminal sample. In aluminium, the residual temperature is $1440 \mathrm{~K}$. If the amount of waste heat exceeds the enthalpy of vaporization (or melting), the terminal sample will be in the vaporised (or molten) state. In peaceful nuclear explosions (e.g., see Chidambaram and Ramanna 1975), when a nuclear explosive is detonated underground in a rock medium, about $20 \%$ of the explosive energy is utilised in vaporising approximately 70 tons of rock, and another $20 \%$ in melting approximately 300 tons of rock for every kton of yield.

\section{Range of shock pressures achieved experimentally}

By keeping chemical explosives (usually in the form of lenses and pads) in contact, shock pressures of a few $100 \mathrm{kbar}$ can be transmitted into materials. Alternately, a metal plate (flyer plate) can be accelerated by a contact explosive or a gas gun, and can subsequently be made to deliver its momentum to a target plate by impact. Pressures up

* $1 \mathrm{Mpa}=10 \mathrm{bar}, 1 \mathrm{Gpa}=10 \mathrm{kbar}, 1 \mathrm{Tpa}=10 \mathrm{Mbar}$. 
to several Mbars can be achieved in this manner, though the pressure pulse widths are smaller (typically a few mm in space); still higher pressures can be obtained using curved rather than plane geometries. Exploding foils and rail guns can also be used to accelerate flyer plates. The highest shock pressures achieved to date have been through laser - or nuclear explosive-driven shocks and are in the range of tens of Mbars (for a more detailed discussion see Godwal et al 1983a). The metallurgical and physical observations to be discussed below have generally been on samples shocked to pressures less than about 1 Mbar.

\section{Finite elastic-plastic deformation by shock waves}

When a plane shock wave propagates through a material, the change in density occurs-from a macroscopic point of view-through uniaxial compression. Consider a volume element of cross-sectional area $A$ (perpendicular to the direction of shock propagation) and length $L$. During shock compression, $A$ remains unchanged while $L$ decreases in direct proportion to the decrease in the specific volume (see figure 2). If the element were allowed to expand from the final compressed state without lateral constraints it would reach normal density at the boundaries shown by the broken lines, provided it had been in a state of hydrostatic strain and stress in the compressed state. This gives us the elastic and plastic strain components in the direction of shock propagation and in the lateral directions, as indicated in the figure.

The time scales involved in shock propagation are typically in the $\mu \mathrm{sec}$ region, while the rise times are well below a nsec. Are conditions of hydrostatic stress and strain achieved behind the shock front, and, if so, how quickly? The answer to this question comes from the beautiful flash x-ray diffraction experiments of Johnson and Mitchell (1980) with single crystals of LiF, Al and graphite. Specific Bragg reflections were recorded using a Mo $\mathrm{x}$-ray pulse synchronised with the duration of the shock compression of the crystal. These authors concluded that: (a) single crystals transform to the hydrostatically-compressed state within less than $20 \mathrm{nsec}$ - there is no immediate conversion to a polycrystalline state; (b) the unit cell volume compression is in agreement with the conventional macroscopic volume compression data to within $1 \%$, independent of crystal orientation (shocks of strengths between $400 \mathrm{kbar}$ and $1 \cdot 1 \mathrm{Mbar}$ were propagated along $[100]$ and $[111]$ in $\mathrm{LiF}$ ). In related experiments at Trombay (Gupta 1980) we shocked polycrystalline samples of zirconium (hexagonal crystal

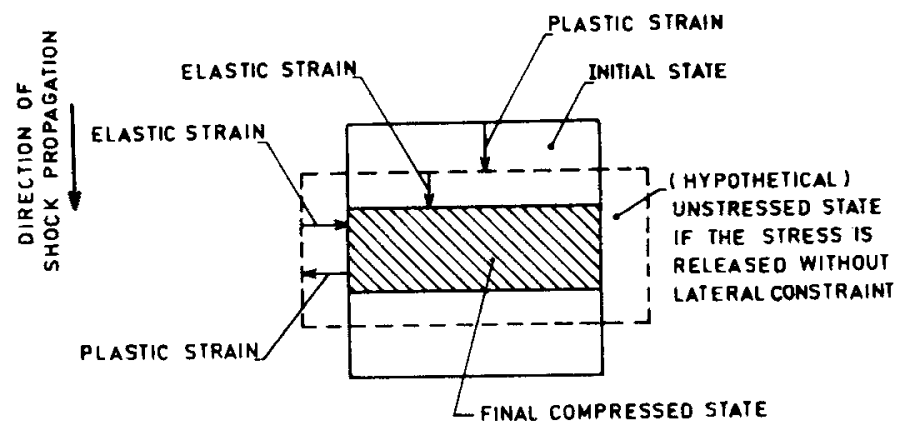

Figure 2. Finite elastic-plastic deformation created by a shock wave. 
system) which showed a prominent texture $(\{100\}$ planes preferentially normal to the axis of the cylindrical sample). The texture was retained to a substantial extent in the terminal sample, after shock loading to about $150 \mathrm{kbar}$.

At very low pressures, the unit cell of the crystal may be expected to respond by onedimensional compression. Correspondingly, in the Hugoniot curve, there is a pressure value known as the Hugoniot elastic limit (HEL) below which the response of the material to the shock pressure is nearly linear; the Hugoniot really begins to curve (concave upwards) from this point. The HEL is related to the shear strength of the material; one may expect that, for shock pressures lower than this limit, no rearrangement of the crystal lattice occurs (unlike in strong shocks discussed below). However, Jamet (1980) found, in some recent flash $\mathrm{x}$-ray experiments with $\mathrm{NaCl}$ single crystals ( $\mathrm{HEL}=280$ bar), that the one-dimensional deformation must be taken into account even for shock pressures above $10 \mathrm{kbar}$. Some experiments on the measurement of triaxial stress components using piezo-resistive gauges for shock pressures below, around and above the HEL are now being carried out for several materials (see, e.g. Chartagnac 1982).

Computer simulation of shock propagation in crystal lattices has also been attempted (Tsai and Macdonald 1976). Shock compression is initiated in a semi-infinite lattice by moving the lattice along the $\mathrm{x}$-axis towards the origin with (mass) average velocity corresponding to the particle velocity $U_{p}$ defined in (1)-(3), and making it collide with its image at $x=0$. Many effects are correctly predicted by these molecular dynamics calculations including the achievement of thermal equilibrium and a state of hydrostatic stress, and also the right temperature. However, the strain in the compressed state turns out to be far from hydrostatic! This is related, we believe, to the computer limitation that necessitates a small-sized crystal lattice without scope for the generation of dislocations. As we shall see, the introduction of mobile-dislocation mechanisms is unavoidable in the description of shock propagation in solids.

\section{Defect-generation during shock propagation}

A variety of point, line and planar defects can be induced by shocks. Solid samples are usually examined after recovery following shock loading, and there is always the question of the relation of the residual defects to those present during the different stages of shock loading. On the one hand, shock heating may anneal out defects; on the other, the shock unloading process may lead to further plastic deformation. The former may be prevented to some extent by doing the experiment at liquid nitrogen temperatures, and then ejecting and storing the sample also at the same low $T$; electrical resistivity annealing measurements in copper shock-loaded (70-380 kbars) in this manner yield typical vacancy concentrations $\sim 10^{-4}$ (Mogilevskii 1970). The vacancy concentrations estimated from active resistance measurements in shock-loaded silver foils are perhaps ten times higher (Dick and Styris 1975; Graham 1981). Similarly, the density of dislocations in metals increases with shock pressure-approximately as $P^{1 / 2}$, according to Murr and Kuhlmann-Wilsdorf (1978). This density is about $10^{10}-10^{11} \mathrm{~cm}^{-2}$ in recovered samples shock-loaded up to $100-200 \mathrm{kbar}$, although once again the dislocation density could be much higher during shock propagation (Graham 1981; Svensson 1981). 
In the original model of Smith (1958) for shock wave propagation, an interface of dislocations was postulated between the shocked and unshocked regions so that the deviatoric stresses arising from the shock could be relieved. The dislocation interface separates two lattices with different parameters. This interface of dislocations would, according to Smith, move with the shock front. If the dislocations move on a slip plane making an angle $\phi$ with the shock-front plane, the velocity of the dislocations becomes $U_{s} \operatorname{cosec} \phi$ which produces the necessary plastic strain. Such supersonic dislocations are, however, unacceptable for several reasons (Meyers and Murr 1981). This had led Meyers to postulate a new model whose essential features are as follows: (a) Dislocations are homogeneously at (or close to) the shock front by the deviatoric stresses set up by the state of uniaxial strain; the generation of these dislocations relaxes the deviatoric stresses. Adjacent dislocation layers are made up of dislocations with Burgers vectors of opposite signs; (b) The dislocations move short distances at subsonic speeds; (c) New dislocation interfaces are generated as the shock wave propagates through the material.

This model qualitatively explains many experimental results. Meyers and Murr also believe that the rarefaction part of the wave (corresponding to shock unloading) plays only a minor role in dislocation generation.

The shock front has a width $W$ extended over a few lattice spacings, i.e., $W \sim 10^{-6} \mathrm{~cm}$. The strain rate $\dot{\varepsilon}$ can be written as (Gilman 1979)

$$
\dot{\varepsilon} \simeq \frac{U_{s}}{2 W} \frac{\left(\rho_{1}-\rho_{0}\right)}{\rho_{0}} .
$$

If the shock velocity is taken to be $1 \mathrm{~cm} / \mu \mathrm{sec}$ and a density compression of 2 is achieved by the shock, $\dot{\varepsilon} \approx 10^{12} \mathrm{sec}^{-1}$, which is enormous. What is the mechanism of the transport of momentum down the steep velocity gradient? It is through atoms and dislocations, and the dislocation velocity may be expected to be proportional to $U_{s}$. The drag pressure $D$, which acts in opposition to the driving pressure $P$ and equals it under steady motion conditions, can be written as a product of an effective viscosity $\eta$ and the strain rate $\dot{\varepsilon}$. Gilman finds that the value of the viscosity that arises from the dislocations is of the order of 1 poise. As both $\eta$ and $\dot{\varepsilon}$ are proportional to $U_{s}, D \sim U_{s}^{2}$, and hence can be very large for strong shocks. I have discussed this aspect in detail to emphasize the complexity that a first-principle calculation of shock wave structure, shock width, etc., would entail. The heating of the material by the shock wave can, however, be calculated using (1)-(3) and condensed matter theory, because the contribution of the various defects produced by the shock wave propagation to the free energy of the material is small.

What about shock propagation in liquids? One has to re-interpret the mobiledislocation mechanisms in this case, taking into account the short-range order in liquids on the one hand, and the narrow width of the shock front on the other. To the best of my knowledge, no work has been done along these lines.

\section{Phase transformations and melting under shock}

Most phase transitions observed under static pressures are also observed under shock pressures. In fact, many sluggish phase transitions are readily observed under shock conditions because the deviatoric shear stress provides a driving force to overcome any 
activation barrier. The phase transformation is best examined in terms of $U_{s}$ and $U_{p}$, rather than the conventional variables $P, V$. The plot of $U_{s}$ vs $U_{p}$ is (surprisingly) linear for a given phase (up to pressures of several megabars), and shows a discontinuity and change of slope upon the occurrence of a phase transformation. There is one important point which must be kept in mind when discussing shock-induced phase transformations: unlike static pressures, shock pressures also induce heating, and the rise in temperature may influence or inhibit the transformation. For example, in the alpha (hcp) to omega (simpic hexagonal) phase transformation in $\mathrm{Ti}$, the amount of $\omega$-phase formed increases continually with pressure in the 'static' case; in contrast, under shock this amount first increases and then decreases as the shock pressure is increased. The reason for this behaviour is the change in temperature (Sikka et al 1982).

As one keeps on increasing the shock pressure, does the shock heating lead to melting in the compressed state, and, if so, what is the melting temperature? There is still considerable controversy with regard to this point. This is because the slope changes in the $U_{s}-U_{p}$ curves are either small or else have an alternative interpretation in terms of certain electronic changes. The shock-Hugoniot measurements show, for example, the existence of a relatively less-compressible high-pressure phase (beyond $300 \mathrm{kbar}$ ) in all members of the rare-earth series. This 'stiffening' of the Hugoniot curves has been identified with melting by Carter et al (1975), but Gust and Royce (1973) attribute it to the onset of a repulsive interaction between the noble-gas atomic cores. McMahan et al (1981), on the other hand, have analysed this phenomenon in terms of the completion of $s-d$ transfer in $\mathrm{La}$. Again, in $\mathrm{Pb}$, the various estimates of shock melting pressures range from $220 \mathrm{kbar}$ to $1.24 \mathrm{Mbar}$ (Duvall and Graham 1977).

The melting temperature under shock compression increases as indicated schematically in figure 3. We feel that the onset of shock melting may be estabiished by a combination of shock temperature measurement and theoretical considerations (Godwal et al 1983b). The Hugoniot curves obtained by equation-of-state calculations in the solid and liquid states are quite distinct in the $T-P$ plane, as shown schematically in figure 3. There is a mixed solid-liquid region in the middle. (We have done these calculations for $\mathrm{Al}$ and $\mathrm{Pb}$ by the pseudopotential method. At $\rho_{1} / \rho_{0}=1.8$ in $\mathrm{Al}$, for example, the temperatures predicted are $12500 \mathrm{~K}$ and $8100 \mathrm{~K}$ respectively using the corresponding solid and liquid state theories.) Thus, a measurement of shock temperatures over a range of shock pressures will presumably yield information of the type shown in figure 3 , so that the onset of melting may be discerned clearly.

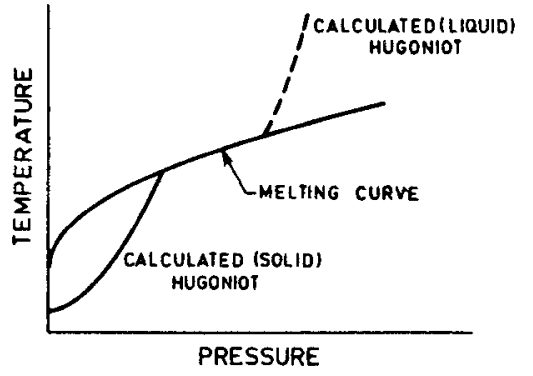

Figure 3. Schematic phase diagram for melting by shock heating. 


\section{Conclusions}

There are many problems in the shock deformation of metals which are not yet fully understood. Most of the metallurgical studies have been on terminal samples shockloaded to a few $100 \mathrm{kbar}$ or less. Large concentrations of a variety of defects are found in these samples. These concentrations are presumably even greater during the various stages of shock propagation, since shock heating is supposed to anneal out many of the defects. The generation and motion of dislocations are essential in explaining shock front propagation which leaves behind a shocked region in a state of essentially hydrostatic strain (at least for strong shocks). Some computer simulation studies on atomistic models have also been attempted, but they do not account for all the experimental facts. Evidence for the melting of materials under very strong shocks is still in some doubt and may need a combination of theory and shock temperature measurement in order to be established conclusively. Much theoretical and experimental work still remains to be done in the field of shock deformation and related effects in materials.

\section{Acknowledgements}

The author thanks Dr S K Sikka and Dr B K Godwal for many helpful discussions.

\section{References}

Carter W J, Fritz J, Marsh S P and McQueen R G 1975 J. Phys. Chem. Solids 36741

Chartagnac P F 1982 J. Appl. Phys. 53948

Chidambaram R and Ramanna R 1975 Proc. Tech. Commun. PNE IV (Vienna: International Atomic Energy Agency) p 421

Dick J J and Styris D L 1975 J. Appl. Phys. 461602

Duvall G E and Graham R A 1977 Rev. Mod. Phys. 49523

Gilman J J 1979 J. Appl. Phys. 504059

Godwal B K, Sikka S K and Chidambaram R 1983a J. Phys. Rep. (under publication)

Godwal B K, Sikka S K and Chidambaram R 1983b (unpublished)

Graham R A 1981 in Shock waves and high-strain-rate phenomena in metals (eds) M A Meyers and L E Murr (New York: Plenum Press) p 375

Gupta S C 1980 Ph.D Thesis, University of Bombay (unpublished)

Gust W H and Royce E B 1973 Phys. Rev. B8 3595

Jamet F 1980 in High pressure science and technology (eds) B Vodar and Ph Marteau (Oxford: Pergamon Press) Vol 2 p 974

Johnson Q and Mitchell A C 1980 in High pressure science and technology (eds) B Vodar and Ph Marteau (Oxford: Pergamon Press) Vol 1 p 977

McMahan A K, Skriver H L and Johanseen B 1981 Phys. Rev. B23 5016

Meyers M A and Murr L E 1981 Shock waves and high strain-rate phenomena in metals (eds) M A Meyers and L E Murr (New York: Plenum Press) p 487

Mogilevskii M A 1970 Combustion, explosion and shock waves Vol 6 p 197

Murr L E and Kuhlmann-Wilsdorf D 1978 Acta Met. 26847

Sikka S K, Vohra Y K and Chidambaram R 1982 Prog. Mater. Sci. 27245

Smith C S 1958 Trans. AIME 212574

Svensson T 1981 Shock waves and high strain rate phenomena in metals (eds) M A Meyers and L E Murr (New York: Plenum Press) p 547

Tsai D H and Macdonald R A 1976 High Temp. High Pressures 8403 


\section{Discussion}

C K Majumdar: Entropy production is not taken into account satisfactorily in any essentially hydrodynamics-based formalism. Eckart's work (1945) may perhaps indicate how this aspect should be incorporated into the description of shocks.

K Srinivasa Raghavan: There is nothing unusual about nonhydrostatic strain due to hydrostatic stress, because one is dealing with nonhomogeneous structures.

R Chidambaram: The question here is whether, in Tsai's molecular dynamics calculation on a bcc crystal, unit cells can be strained nonhydrostatically under hydrostatic stress. This has been achieved here by 'buckling' of neighbouring cellsakin to twinning. This picture appears to be somewhat artificial to me, the problem arising from the neglect of mechanisms of plastic deformation.

K A Padmanabhan: Can the amount of explosive charge be estimated, when, say, a hollow cylinder of $\mathrm{Al}$ is cald on to a hollow inner cylinder of steel?

Chidambaram: The attenuation behind the shock front is related to the ratio of the mass of the metal to the mass of the explosive. In the problem of explosive welding one has to strike a balance between having too little explosive (the attenuation is then too high) and having too much explosive (the metal cylinder is then damaged).

A P Pathak: Can channeling by charged particles be used to extract information about the defects while experiments on mechanical properties (including shock wave propagation) are in progress?

Chidambaram: I think the penetration of charged particles into the specimen could pose problems, as the diameter of the metal plates under study $\gtrsim 1 \mathrm{~cm}$

R Krishnan: In uniaxial tensile testing, an increased strain rate is equivalent to a reduction in test temperature. Is this so also for hydrostatic stress?

Chidambaram: For shock induced deformation, a higher strain rate implies a higher shock pressure which would lead to increased shock heating.

$S$ Ramaseshan: How long does the rise in $T$ due to the shock wave take to cool down?

Chidambaram: The fall from the high temperature in the compressed state to the residual temperature after shock unloading usually occurs in $\mu \mathrm{sec}$. The cooling beyond that is by normal mechanisms.

Ramaseshan: How are boundary conditions specified in the molecular dynamics study of the shock front? How does this method differ from the finite element approach?

Chidambaram: As in usual solid state theory, cyclic boundary conditions were used by Tsai and Macdonald. The finite element method is a more general mathematical technique that is useful even in problems where there are no periodic lattices.

P Rodriguez: What is an ideal powder?

Chidambaram: In the context of diffraction, it is one in which there is no preferred orientation of the grains.

Rodriguex: For melting to occur, the dislocation density must be of the order 
$10^{13} / \mathrm{cm}^{2}$, but experimentally it is $10^{11} / \mathrm{cm}^{2}$. How is this discrepancy explained?

Chidambaram: The high temperatures prevailing probably cause annealing of some of the dislocations, reducing their density.

Rodriguez: The high strain rates used in shock experiments are also used in metallurgical techniques like explosive forming and welding. The physical basis of these methods needs to be understood.

Rodriguez: In the flyer plate method used for welding and forming the interface is wavy. Could you comment on this?

Chidambaram: The uniformity with which one can produce a plane wave or shock wave by any of these methods is about $100 \mathrm{nsec}$. Within this time a non-uniformity of the order of $0.1-1 \mathrm{~mm}$ sets in. This has to be carried through.

D Kumar: In the energy diagram, a part of the triangle is the recoverable elastic energy and a part is the dissipated heat energy. Does the rest go into the formation of dislocations?

Chidambaram: The energy required to create dislocations is small and is generally ignored in the book-keeping.

S N Bandopadhyay: What is the evidence that the strain is hydrostatic?

Chidambaram: Flash x-ray diffraction experiments on cubic crystals indicate that the crystal behind the shock front reaches a state of hydrostatic strain within 20 nsec. 